\title{
Chemical evolution of D in the Local Disk
}

\author{
Takuji Tsujimoto ${ }^{1}$ and Joss-Bland-Hawthorn ${ }^{2}$ \\ ${ }^{1}$ National Astronomical Observatory, Mitaka-shi, Tokyo 181-8588, Japan \\ email: taku.tsujimoto@nao.ac.jp \\ ${ }^{2}$ Institute of Astronomy, School of Physics, University of Sydney, NSW 2006, Australia
}

\begin{abstract}
Chemical features of the local disk have firmly established the picture for the formation of the Galactic disk that the star formation has proceeded under the continuous accretion of low-metallicity gas from the halo. It sets two determinant processes for the evolution of deuterium (D), that is, the destruction of $\mathrm{D}$ in the interior of stars and the supply of new (nearly) primordial D associated with the gas infall. Conventional Galactic chemical evolution (GCE) models predict that this scheme leads to a monotonic decrease in $\mathrm{D} / \mathrm{H}$ with time and ends up in the present-day $\mathrm{D} / \mathrm{H}$ abundance $(\mathrm{D} / \mathrm{H})_{0}$ which is severely lower than the recently observed estimates. These predicted features are the natural results of a construction of the metal-rich ( solar abundance) local star+gas system. Here we propose that the new GCE models, that incorporate large-scale winds form the Galactic bulge which entrain heavy elements and drop them on the disk with the recent tendency of star formation in tune with the observed implications, make the system rich in both metals and D. In addition, our finding of a gradual increase in $\mathrm{D} / \mathrm{H}$ with time during the last several Gyr is observationally supported by the $\mathrm{D} / \mathrm{H}$ abundance for the protosolar cloud lower than $(\mathrm{D} / \mathrm{H})_{0}$.
\end{abstract}

Keywords. Galaxy: disk, evolution — Stars: abundances

Recent Far-Ultraviolet Spectroscopic Explorer (FUSE) observations reveal that the present-day $\mathrm{D} / \mathrm{H}$ abundance $(\mathrm{D} / \mathrm{H})_{0}$ for interstellar matter (ISM) in the Galactic disk is surprisingly as high as $\geqslant 2.31 \pm 0.24 \times 10^{-5}$ close to the primordial $(\mathrm{D} / \mathrm{H})_{p}$ after correcting for dust depletion (Linsky et al. 2006). The high $\mathrm{D} / \mathrm{H}$ abundance of $2.2_{-0.6}^{+0.8} \times 10^{-5}$ in the warm neutral medium of the lower Galactic halo, which originates in the disk and is elevated into the halo (Savage et al. 2007), strongly supports the claim by Linsky et al.

The difference between $(\mathrm{D} / \mathrm{H})_{p}$ and $(\mathrm{D} / \mathrm{H})_{0}$ is defined as a deuterium astration factor $f_{d}=(\mathrm{D} / \mathrm{H})_{p} /(\mathrm{D} / \mathrm{H})_{0}$. The value of $f_{d}$ should absolutely be $>1$, because it is believed that all $\mathrm{D}$ are produced during Big Bang nucleosynthesis, and only the destruction process of $\mathrm{D}$ occurs in the interior of stars in the post-Big Bang evolution. Thus, the value of $f_{d}$ is determined by the fraction of matter which has never been cycled through stars in the present ISM. Accordingly, $f_{d}$ is closely related to the enrichment degree by heavy elements in the Galaxy evolution through star formation that destroys D while fresh heavy elements are released into the ISM when the stars die. In the end, due to the fact that the D abundance in the ISM is anti-correlated with the metallicity in the system, the well-enriched ( $\sim$ solar metallicity) local disk demands a high $f_{d}$. Indeed, the standard GCE models predict $f_{d}=1.39-1.83$ for the local disk (Romano et al. 2006). In other words, the local abundances of heavy elements such as Fe or $\alpha$-elements in the ISM and long-lived stars imply $f_{d} \approx 1.4$ at the least, that is equivalent to $(\mathrm{D} / \mathrm{H})_{0}<2.0 \times 10^{-5}$. On the other hand, the high $(\mathrm{D} / \mathrm{H})_{0}$ implied by the recent observations means a smaller $f_{d}$. For the estimates of $(\mathrm{D} / \mathrm{H})_{p}=2.75 \times 10^{-5}$ (Cyburt et al. 2003) and $(\mathrm{D} / \mathrm{H})_{0}=2.31 \times 10^{-5}$, we obtain $f_{d}=1.19$, which is incompatible with the theoretical prediction.

The $\mathrm{D} / \mathrm{H}$ abundances which bridge between the primordial $(\mathrm{D} / \mathrm{H})_{p}$ and the presentday $(\mathrm{D} / \mathrm{H})_{0}$ give a crucial information on the evolution of $\mathrm{D}$. Viewing from this angle the 


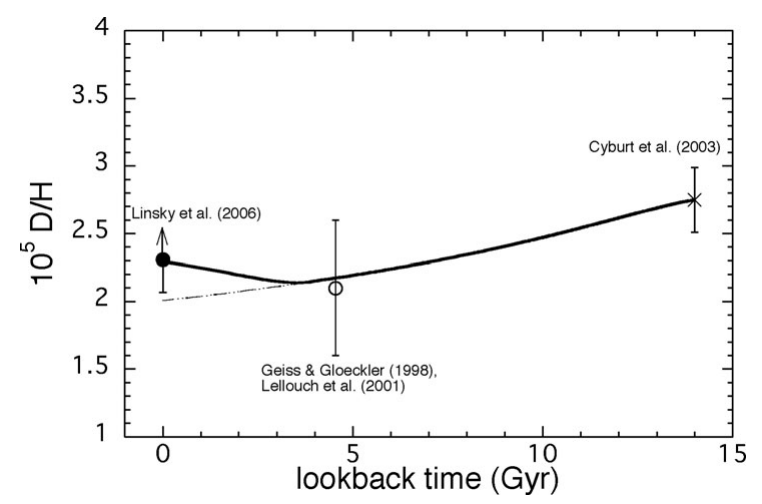

Figure 1. The evolution of $\mathrm{D} / \mathrm{H}$ abundance in the local disk predicted by the wind+decreasing SFR model (solid line). For reference, the model case with a constant SFR coefficient is shown by the dashed line. Three observed points are for the primordial value (Cyburt et al. 2003), the value for the protosolar cloud (Geiss \& Gloecker 1998, Lellouch et al. 2001), and the local ISM value (Linsky et al. 2006).

$\mathrm{D} / \mathrm{H}$ in the protosolar cloud, which represents the abundance at the age of $\sim 4.6 \mathrm{Gyr}$ in the Galaxy, the obtained results are quite intriguing. The $\mathrm{D} / \mathrm{H}$ abundances deduced from the atmosphere of jupiter (Lellouch et al. 2001) or from the solar wind (Geiss \& Gloeckler 1998) are $2.1 \pm 0.4(0.5) \times 10^{-5}$. If these estimates are correct, does it imply that $\mathrm{D}$ in the local disk has gradually increased during the last several Gyr?

Recent several works reveal the star formation history in the local disk, claiming that the star formation rate (SFR) has been clearly declining for the last several Gyr (Fuchs et al. 2009). In addition, recent new view on chemical evolution of the Galactic disk has suggested that large-scale winds from the Galactic bulge, which entrain a large amount of heavy elements, enrich the disk (Tsujimoto 2007). Theoretical framework that the Galaxy disk has evolved through an ongoing infall and the winds, together with the renewed constraint from the star formation history in the local disk, will open a new channel for the interpretation of the $\mathrm{D}$ evolution in the local disk.

The predicted time-evolution of $\mathrm{D} / \mathrm{H}$ in this scheme is shown in Figure 1 . Here the primordial $(\mathrm{D} / \mathrm{H})_{0}$ is set to be $2.75 \times 10^{-5}$. From its initial value, the $\mathrm{D} / \mathrm{H}$ abundance starts to decrease with time, and subsequently its change turns to a gradual increase around 4 Gyr ago. This turnover results from the supply of primordial D by an infall that overwhelms the destruction of $\mathrm{D}$ through the star formation owing to its low rate. Then, it finally enables to achieve a present-day high $\mathrm{D} / \mathrm{H}$ abundance of $2.3 \times 10^{-5}$, fully consistent with the observed value, after passing $\mathrm{D} / \mathrm{H} \sim 2.1 \times 10^{-5}$ equivalent to the protosolar value around $4.6 \mathrm{Gyr}$ ago. For reference, the case with a constant SFR over the full age is indicated by dashed line.

\section{References}

Cyburt, R. H., Fields, B. D., \& Olive, K. A. 2003, Phys. Lett. B, 567, 227

Fuchs, B., Jahreiß, H., \& Flynn, C. 2009, AJ, 137, 266

Geiss, J. \& Gloeckler, G. 1998, Space Sci. Rev., 84, 239

Lellouch, E. et al. 2001, A\&A, 670, 610

Linsky, J. L. et al. 2006, ApJ, 647, 1106

Romano, D., Tosi, M., Chiappini, C., \& Matteucci, F. 2006, MNRAS, 369, 295

Savage, B. D., Lehner, N., Fox, A., Wakker, B., \& Sembach, K. 2007, ApJ, 659, 1222

Tsujimoto, T. 2007, ApJ, 665, L115 


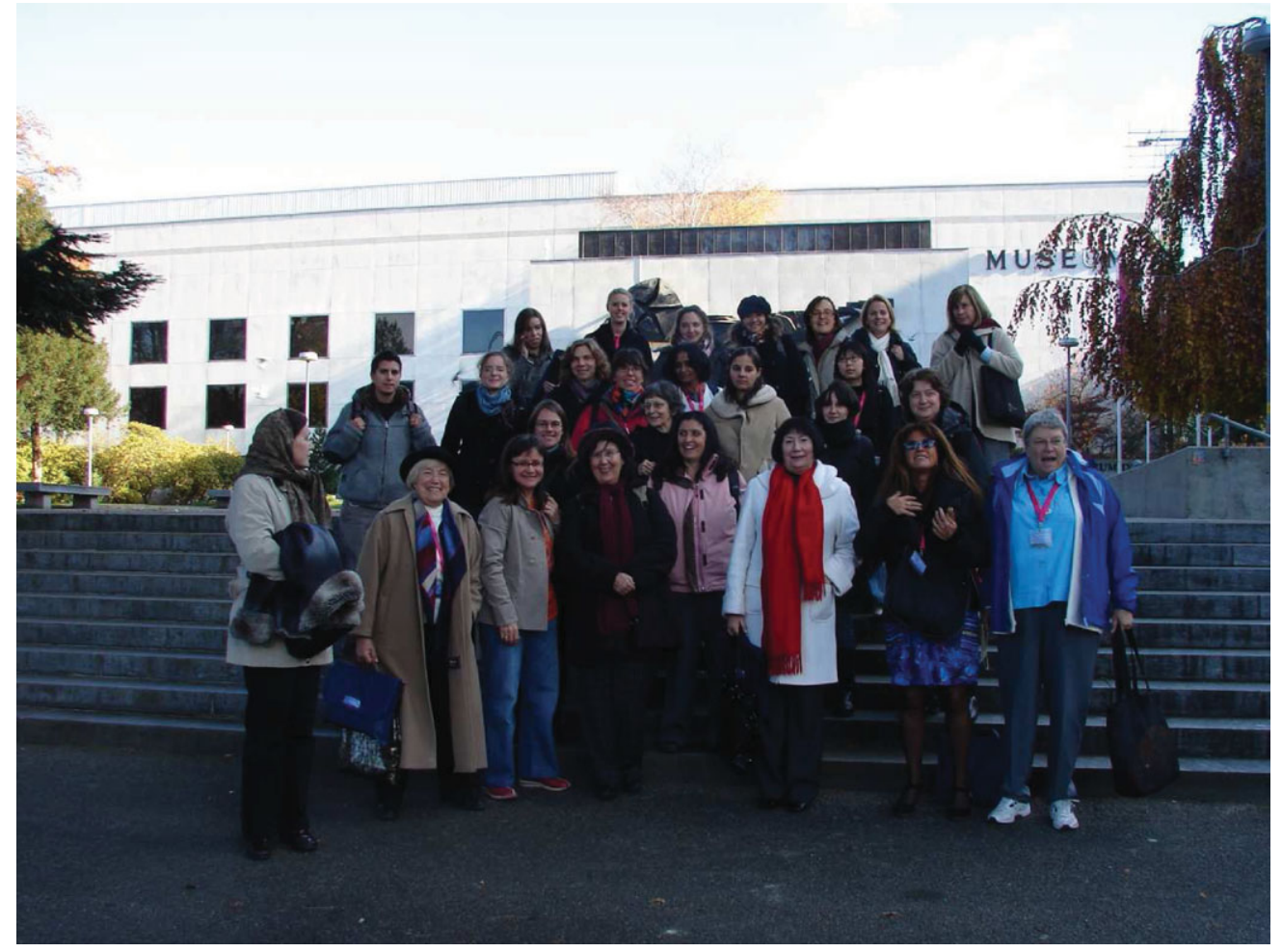

IAU 268 women in front of the Museum of Natural History

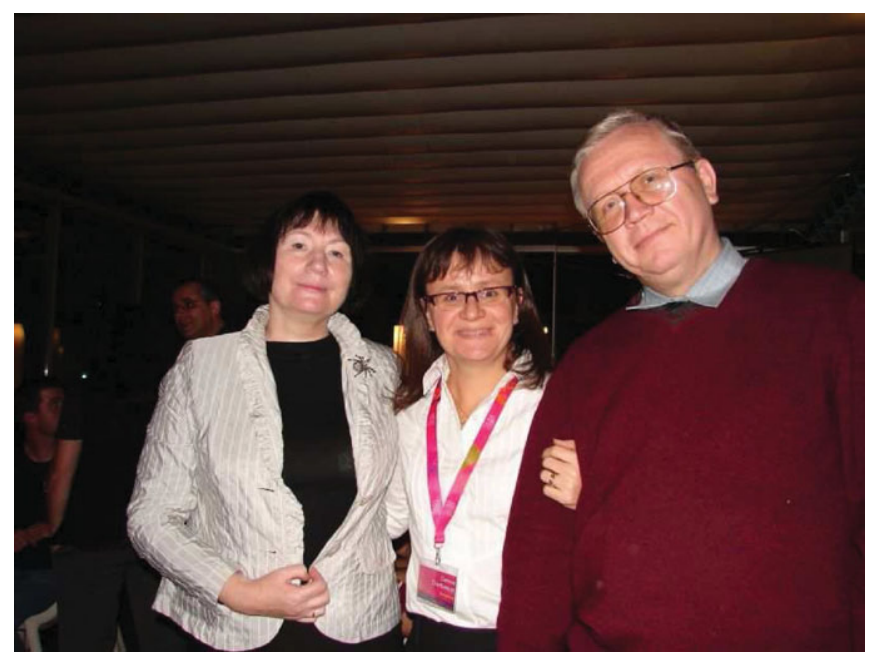

Tamara Mishenina, Corinne Charbonnel, Yuri Izotov 


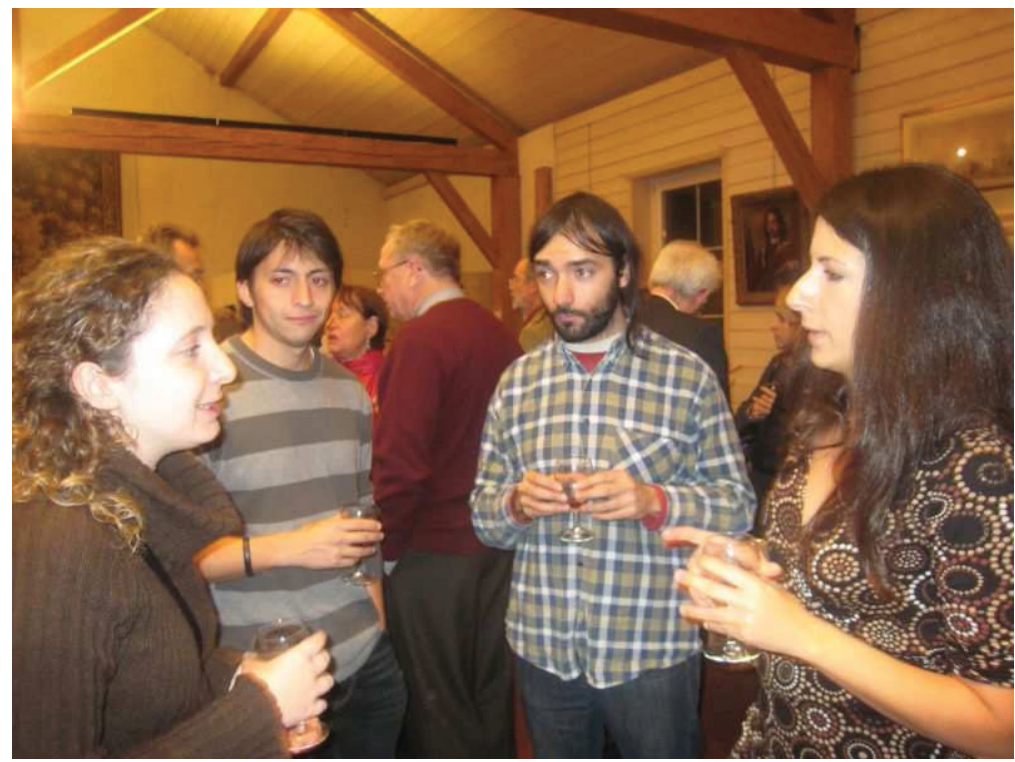

Anna Faviola Marino, Oscar Gonzalez, Antonino Milone, Marcella di Criscienzo

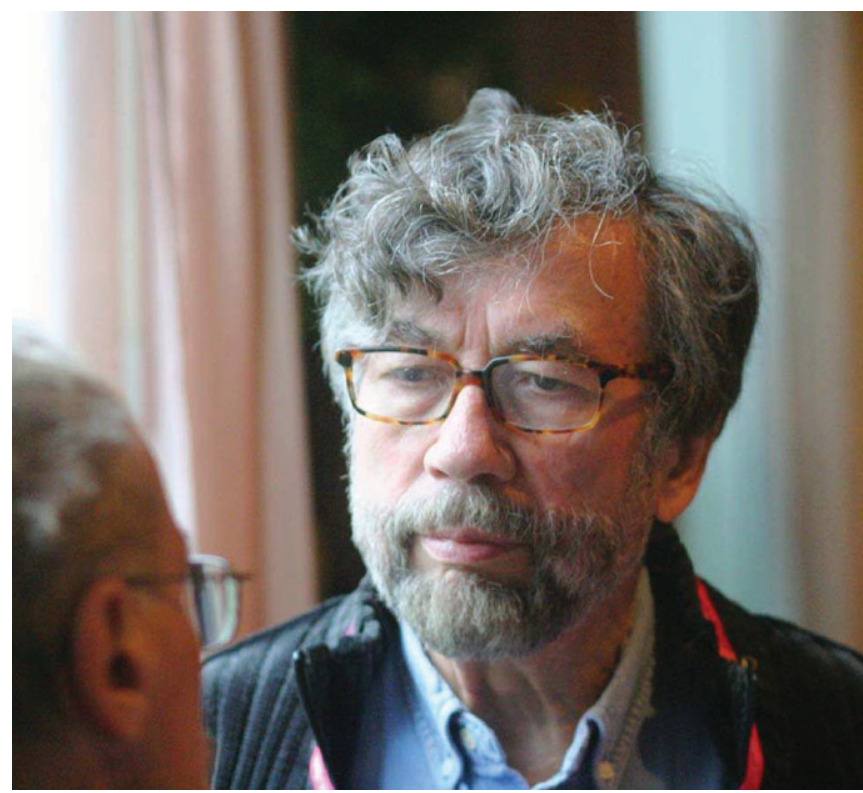

Jean-Paul Zahn 